\title{
Immune landscape of advanced gastric cancer tumor microenvironment identifies immunotherapeutic relevant gene signature
}

Simeng Zhang ${ }^{1,2,3,4}$, Mengzhu Lv ${ }^{5}$, Yu Cheng ${ }^{1,2,3,4}$, Shuo Wang ${ }^{1,2,3,4}$, Ce Li $^{1,2,3,4}$ and Xiujuan Qu $u^{1,2,3,4^{*}}$

\begin{abstract}
Background: Advanced gastric cancer (AGC) is a disease with poor prognosis due to the current lack of effective therapeutic strategies. Immune checkpoint blockade treatments have shown effective responses in patient subgroups but biomarkers remain challenging. Traditional classification of gastric cancer (GC) is based on genomic profiling and molecular features. Therefore, it is critical to identify the immune-related subtypes and predictive markers by immuno-genomic profiling.

Methods: Single-sample gene-set enrichment analysis (sSGSEA) and ESTIMATE algorithm were used to identify the immue-related subtypes of AGC in two independent GEO datasets. Weighted gene co-expression network analysis (WGCNA) and Molecular Complex Detection (MCODE) algorithm were applied to identify hub-network of immunerelated subtypes. Hub genes were confirmed by prognostic data of KMplotter and GEO datasets. The value of hubgene in predicting immunotherapeutic response was analyzed by IMvigor210 datasets. MTT assay, Transwell migration assay and Western blotting were performed to confirm the cellular function of hub gene in vitro.
\end{abstract}

Results: Three immune-related subtypes (Immunity_H, Immunity_M and Immunity_L) of AGC were identified in two independent GEO datasets. Compared to Immunity_L, the Immuntiy_H subtype showed higher immune cell infiltration and immune activities with favorable prognosis. A weighted gene co-expression network was constructed based on GSE62254 dataset and identified one gene module which was significantly correlated with the Immunity_H subtype. A Hub-network which represented high immune activities was extracted based on topological features and Molecular Complex Detection (MCODE) algorithm. Furthermore, ADAM like decysin 1 (ADAMDEC1) was identified as a seed gene among hub-network genes which is highly associated with favorable prognosis in both GSE62254 and external validation datasets. In addition, high expression of ADAMDEC1 correlated with immunotherapeutic response in IMvigor210 datasets. In vitro, ADAMDEC1 was confirmed as a potential protein in regulating proliferation and migration of gastric cancer cell. Deficiency of ADAMDEC1 of gastric cancer cell also associated with high expression of PD-L1 and Jurkat T cell apoptosis.

Conclusions: We identified immune-related subtypes and key tumor microenvironment marker in AGC which might facilitate the development of novel immune therapeutic targets.

Keywords: Advanced gastric cancer, Tumor microenvironment, Immuno-genomic profiling, WGCNA, ADAMDEC1

\footnotetext{
*Correspondence: xiujuanqu@yahoo.com

1 Department of Medical Oncology, the First Hospital of China Medical

University, 110001 Shenyang, China

Full list of author information is available at the end of the article
} 


\section{Background}

Gastric cancer (GC) is the third leading cause of cancer death and the fifth most common malignancy worldwide [1]. Due to the lack of effective screening to detect early-stage GC, most patients are diagnosed with AGC which has poor prognosis [2]. Although improved therapeutic strategies have been developed, clinical outcomes remain unsatisfactory. Recently, immune checkpoint blockades such as anti-programmed cell-death protein 1(PD1) or programmed cell-death 1 ligand 1 (PD-L1) drugs have been widely used and achieved efficacy in different cancer types [3, 4]. Anti-PD-1/PD-L1 therapy has also been shown to be effective and is approved for third-line treatment in metastatic GC [5]. However, only some subsets of GC patients benefit from immunotherapy. PD-L1 expression, Epstein-Barr infection and microsatellite status have been reported to be associated with immunotherapeutic responsiveness [6, 7]. However, the dominant population and prognostic marker of immunotherapies are currently unknown.

The tumor immune microenvironment (TME) consisting of immune and stromal cells has been proven to be associated with clinical outcomes to immunotherapy $[8,9]$. In GC, the immune microenvironment has a complex relationship with cancer occurrence and progression, which could be regulated by Tumor-infiltrating immune cells (TIICs) $[10,11]$. An increasing number of studies have indicated that TIICs play important roles as prognostic markers and are potential therapeutic targets [12, 13]. The Estimation of Stromal and Immune Cells in Malignant Tumors using Expression data (ESTIMATE) algorithm which is based on single sample gene set enrichment analysis, evaluated the immune and stromal infiltration level in tumors by calculating immune, tumor purity and stromal scores [14]. Recently, the ESTIMATE algorithm has been wildly used to investigate the TME in acute myeloid leukemia, colorectal cancer and breast cancer [15-17]. However, the roles of TIICs in AGC and hub-genes correlations with the TME remain to be fully understood.

In this study, AGC was classified into three distinct subtypes by immuno-genomic profiling and confirmed the reliability of classification model in two independent Gene Expression Omnibus (GEO) datasets. We further identified the hub genes and critical pathways in different immune subtypes of AGC. The results may offer novel evidence in predicting prognosis and immunotherapeutic targets of AGC.

\section{Methods}

Data collection

Microarray data of GSE62254 and GSE29272 were obtained from the Gene Expression Omnibus (www.ncbi. nlm.nih.gov/geo/). The data of GSE62254 was based on GPL570 platforms (Affymetrix Human Genome U133 Plus 2.0 Array, 300 GC patients). The GSE29272 data was based on GPL96 platforms which included 268 GC patients. Two hundred ninety-five samples of GSE62254 and 126 samples of GSE29272 with both gene expression data and clinical parameters of advanced gastric cancer were included. Kaplan Meier-plotter (KMplotter) (http:// www.kmplot.com/) was used for external validation.

\section{Data clustering and evaluation of immune and stromal scores}

Single-sample gene-set enrichment analysis (ssGSEA) was used to evaluate the enrichment levels of the 29 immune signatures and hierarchical clustering was performed according to the ssGSEA score [18, 19]. ESTIMATE algorithm which integrated in "estimate" $\mathrm{R}$ package in $\mathrm{R}$ version 3.6.2. was applied to measure immune microenvironment infiltration based on gene expression data [14].

\section{Proportions of immune cell subsets between GC immune subtypes}

CIBERSORT [20] was used to estimate the proportions of 22 immune cell subsets and the relative expression of 22 immune cell subsets in each sample was determined. $P<0.05$ was set as criteria for subsequent analysis.

\section{Gene-set enrichment analysis}

Gene-set enrichment analysis of each GEO datasets was applied to identify the Gene Ontology (GO) terms and Kyoto Encyclopedia of Genes and Genomes (KEGG) pathways participating in high immunity subgroup of advanced gastric cancer. FDR $<0.05$ was set as criteria to select significance pathways.

\section{Identification of immune subtype-specific genes in advanced gastric cancer}

Weighted Gene Co-expression Network Analysis (WGCNA) was used to construct gene co-expression network and extract the gene information in each module [21]. The correlation between module eigengenes and immune subtypes was evaluated by Pearson's correlation tests. To explore the potential biological process of genes within the immune-related modules, GO and KEGG enrichment analysis were performed and visualized by clusterprofiler package in $R$ project [22]. 
Table 1 Characteristics of GSE62254 and GSE29272 cohort

\begin{tabular}{|c|c|c|c|}
\hline \multicolumn{2}{|l|}{ GSE62254 } & \multicolumn{2}{|l|}{ GSE29272 } \\
\hline Characteristic & $\begin{array}{l}\text { Number of } \\
\text { Patients (\%) }\end{array}$ & Characteristic & $\begin{array}{l}\text { Number of } \\
\text { Patients (\%) }\end{array}$ \\
\hline Age (years) & & Age (years) & \\
\hline Median (Range) & $63(24-86)$ & Median (Range) & $59(23-71)$ \\
\hline Gender & & Gender & \\
\hline Male & $195(66.1 \%)$ & Male & 99 (78.6\%) \\
\hline Female & $100(33.9 \%)$ & Female & $27(21.4 \%)$ \\
\hline T stage & & TNMstage & \\
\hline $\mathrm{T} 2$ & $184(62.4 \%)$ & I & $5(4.0 \%)$ \\
\hline T3 & 90 (30.5\%) & $\|$ & $5(4.0 \%)$ \\
\hline T4 & $21(7.1 \%)$ & III & $108(85.7 \%)$ \\
\hline N stage & & IV & $8(6.3 \%)$ \\
\hline No & $38(12.9 \%)$ & & \\
\hline N1 & $128(43.4 \%)$ & & \\
\hline N2 & 79 (26.8\%) & & \\
\hline N3 & $50(16.9 \%)$ & & \\
\hline \multicolumn{4}{|l|}{ M stage } \\
\hline MO & $268(90.8 \%)$ & & \\
\hline M1 & $27(9.2 \%)$ & & \\
\hline \multicolumn{4}{|l|}{ TNMstage } \\
\hline I & $30(10.2 \%)$ & & \\
\hline$\|$ & 94 (31.9\%) & & \\
\hline III & 95 (32.2\%) & & \\
\hline IV & $76(25.8 \%)$ & & \\
\hline \multicolumn{4}{|l|}{ Lauren } \\
\hline Intestinal & $144(48.8 \%)$ & & \\
\hline Diffuse & $134(45.4 \%)$ & & \\
\hline Mixed & 17 (5.8\%) & & \\
\hline
\end{tabular}

Protein-protein interaction network construction

Protein-protein interaction network (PPI) was constructed by Cytoscape software (v3.6.1) [23]. The hub network was selected by topological features. MCODE (Molecular Complex Detection) algorithm was used to further identify the hub genes in the PPI network [24].

\section{Genomic and clinical data with immunotherapy}

The value of ADAMDEC1 in predicting immunotherapeutic response was analyzed by IMvigor210 datasets. The expression profile and clinical parameter of IMvigor210 dataset that is available under the Creative Commons 3.0 license was downloaded from http://resea rch-pub.gene.com/IMvigor210CoreBiologies. A total of 298 urothelial cancer samples with both gene expression and immune response parameter were selected to further analysis.

\section{Survival analysis}

Survival curves were plotted by the Kaplan-Meier (KM) method and compared with the log-rank test. The expression level of hub-genes was separated to high and low expression according to the median value. $P<0.05$ considered as a threshold to identify the significance genes associated with prognosis of advanced gastric cancer.

\section{Experimental validation \\ Cell culture}

MGC803 gastric cancer cell line was obtained from the Type Culture Collection of the Chinese Academy of Sciences (Shanghai, China). Jurkat $\mathrm{T}$ cells were obtained from the American Type Culture Collection (ATCC, Rockville, MD, USA). All cells were grown in RPMI-1640

Table 2 Clinical Characteristics of Immune Subtypes

\begin{tabular}{|c|c|c|c|c|c|c|c|c|c|c|c|c|c|}
\hline \multicolumn{7}{|c|}{ GSE62254 } & \multicolumn{7}{|c|}{ GSE29272 } \\
\hline & \multicolumn{2}{|c|}{ Immunity L } & \multicolumn{2}{|c|}{ Immunity M } & \multicolumn{2}{|c|}{ Immunity $\mathrm{H}$} & & \multicolumn{2}{|c|}{ Immunity L } & \multicolumn{2}{|c|}{ Immunity M } & \multicolumn{2}{|c|}{ Immunity $\mathrm{H}$} \\
\hline & No. & $\%$ & No. & $\%$ & No. & $\%$ & & No. & $\%$ & No. & $\%$ & No. & $\%$ \\
\hline Age & & & & & & & Age & & & & & & \\
\hline$<60$ & 10 & $9.4 \%$ & 79 & $74.5 \%$ & 17 & $16.0 \%$ & $<60$ & 11 & $16.4 \%$ & 41 & $61.2 \%$ & 15 & $22.4 \%$ \\
\hline$\geq 60$ & 22 & $11.6 \%$ & 140 & $74.1 \%$ & 27 & $14.3 \%$ & $\geq 60$ & 7 & $11.9 \%$ & 48 & $81.4 \%$ & 4 & $6.8 \%$ \\
\hline Gender & & & & & & & Gender & & & & & & \\
\hline Male & 24 & $12.3 \%$ & 143 & $73.3 \%$ & 28 & $14.4 \%$ & Male & 14 & $14.1 \%$ & 70 & $70.7 \%$ & 15 & $15.2 \%$ \\
\hline Female & 8 & $8.0 \%$ & 76 & $76.0 \%$ & 16 & $16.0 \%$ & Female & 4 & $14.8 \%$ & 19 & $70.4 \%$ & 4 & $14.8 \%$ \\
\hline pStage & & & & & & & pStage & & & & & & \\
\hline$|/| \mid$ & 14 & $11.3 \%$ & 93 & $75.0 \%$ & 17 & $13.7 \%$ & $|/| \mid$ & 2 & $20.0 \%$ & 8 & $80.0 \%$ & 0 & $0.0 \%$ \\
\hline III/IV & 18 & $10.5 \%$ & 126 & $73.7 \%$ & 27 & $15.8 \%$ & III/IV & 16 & $13.8 \%$ & 81 & $69.8 \%$ & 19 & $16.4 \%$ \\
\hline \multicolumn{14}{|c|}{ Metastasis } \\
\hline Yes & 16 & $11.3 \%$ & 110 & $77.5 \%$ & 16 & $11.3 \%$ & & & & & & & \\
\hline No & 16 & $10.5 \%$ & 109 & $71.2 \%$ & 28 & $18.3 \%$ & & & & & & & \\
\hline
\end{tabular}


(GibcoBRL, USA) supplemented with 10\% fetal bovine serum (FBS), penicillin $(100 \mathrm{U} / \mathrm{mL})$ and streptomycin $(100 \mathrm{mg} / \mathrm{mL})$, in a humid atmosphere containing $5 \% \mathrm{CO}$ 2 at $37^{\circ} \mathrm{C}$.

\section{Reagents and antibodies}

Anti-ADAMDEC1 antibodies were obtain form Novus Biologicals (USA). Antibodies specific to PD-L1 (13684S) was from Cell Signaling Technology (Danvers, MA, USA). All the other antibodies were purchased from Santa Cruz Biotechnology (USA).

\section{Small interfering RNA (siRNA) transfections}

The ADAMDEC1 siRNA sequences from Beijing GeneX Health technology Co., Ltd. (Beijing, China), were used: 5'-GCCTGTACTTTGGCTCATTGTTCTT-3'. The siRNAs were transfected with Lipofectamine 2000 (Invitrogen, Carlsbad, CA) per the manufacturer's instructions.

\section{MTT assay, Transwell migration assay and Western blotting}

MTT assay, migration assay and Western blot and were performed as described previously [25].

\section{Real-time PCR analysis}

The real-time PCR conditions included initial activation at $95^{\circ} \mathrm{C}$ for $5 \mathrm{~min}$, followed by $15 \mathrm{~s}$ of 45 cycles at $95^{\circ} \mathrm{C}$ and $60^{\circ} \mathrm{C}$ for $1 \mathrm{~min}$ (Applied Biosystems ${ }^{\circledR} 7500$ Real-Time PCR Systems, Thermo fisher, IL, USA). Primer sequences for PD-L1: Forward (5'-TTTCAATGTGACCAGCAC $\left.-3^{\prime}\right)$, Reverse (5'- GGCATAATAAGATGGCTC-3'); 18S: Forward (5'-CCC GGG GAG GTAGTG ACG AAA AAT$\left.3^{\prime}\right)$, Reverse (5'-CGCCCGCCCGCTCCCAAGAT-3').

\section{Cell apoptosis assay}

Jurkat $\mathrm{T}$ cells $\left(3 \times 10^{5} /\right.$ well $)$ were co-cultured with MGC803 cells for $48 \mathrm{~h}$. After that, Jurkat T cells were harvested and stained using an Annexin V-fluorescein isothiocyanate/propidium iodide apoptosis detection kit (BMS500FI-100; Invitrogen; Thermo Fisher Scientific, Inc.) and the number of apoptotic $\mathrm{T}$ cells was determined by FACSCalibur flow cytometry (BD Biosciences, San Jose, CA, USA) according to the protocol. The samples were selected and analyzed by BD Accuri C6.

\section{Statistical analysis}

Data are reported as means \pm SD. Student's t-test or one-way ANOVA were applied to evaluate differences between or among groups. $P<0.05$ was determined statistically significant. Each experiment was repeated at least three times.

\section{Results}

Identification of immune subtypes in AGC by immuno-genomic profiling

AGC data were obtained from GSE62254 and GSE29272 datasets. Samples with both gene expression data and clinical parameters were selected for further analysis (Table 1). To quantify the enrichment of immune cells and pathways in each GC sample, we analyzed 29 immune-related gene sets (Table S1) and calculated ssGSEA scores. The results of hierarchical cluster showed three clusters that were separated in the GSE62254 and GSE29272 datasets (Fig. 1). According to the ssGSEA score, clusters were defined as Immunity Low (Immunity_L), Immunity Medium (Immunity_M)

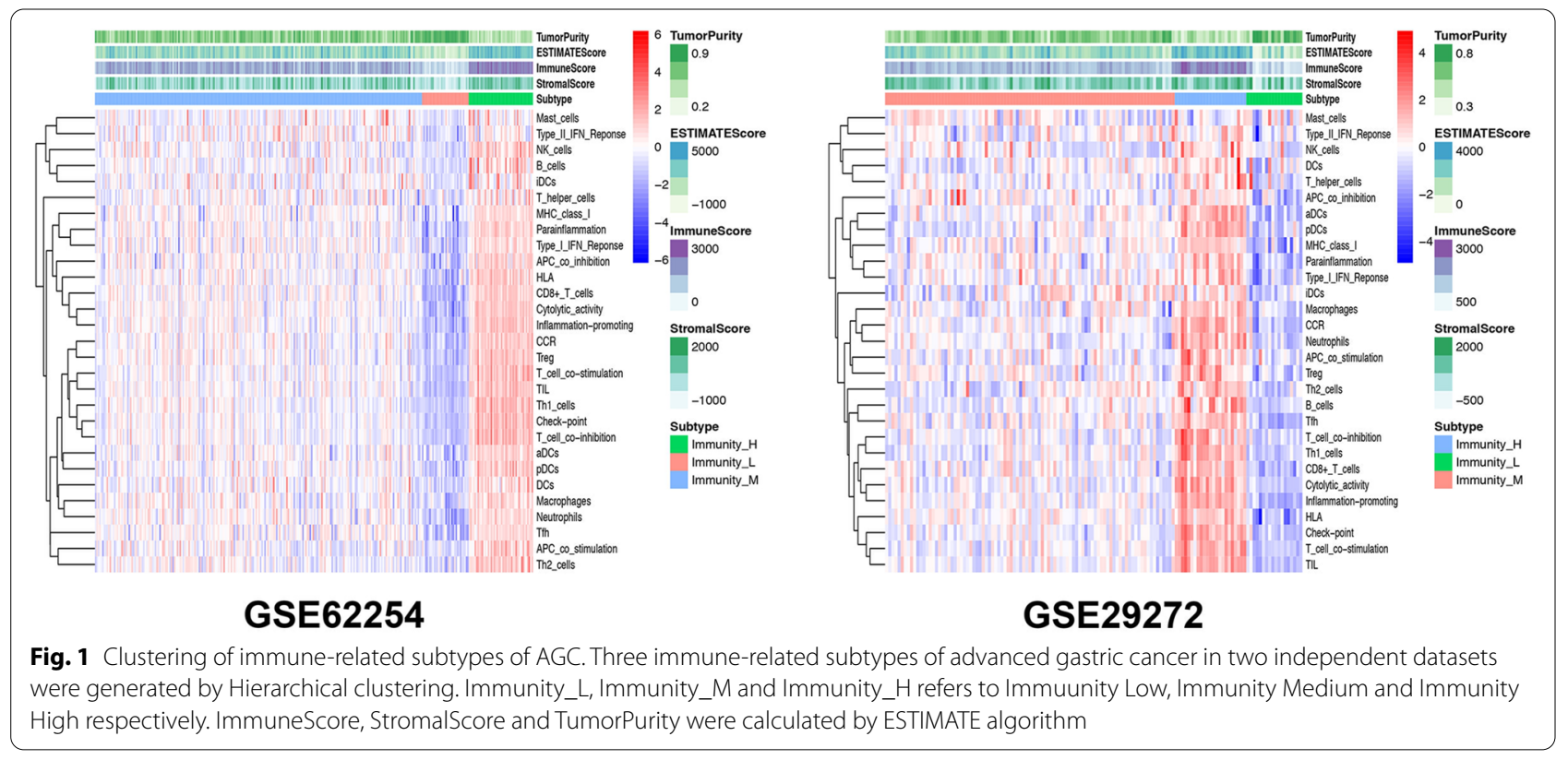


and immunity High (Immunity_H) (Table 2). Using the ESTIMATE algorithm, we calculated immune scores and tumor purity for all samples. Compared to Immunity_L subtype, the immune scores were higher in the Immunity_H (Fig. 2A) subgroup and similar trends were obtained in stromal score in GSE62254 (Additional file 1: Fig. S1A). In contrast, tumor purity scores were lower in the Immunity_H subgroup. These results indicated that immune cells were highly infiltrative in the Immunity_H subgroup and tumor cells were more detected in the Immunity_L subgroup.

Association between the HLA genes and immune subtypes was further investigated. Interestingly, the expression level of HLA genes was notably higher in the Immunity_H compared to the Immunity_L (ANOVA test, $P<0.05$ ) (Fig. 2B). Furthermore, we evaluated proportions of the 22 TIICs in each sample using CIBERSORT plug-in in R package [20]. The fractions of M1 Macrophages, activated memory CD4 $\mathrm{T}$ cells, CD8 $\mathrm{T}$ cells and $\gamma \delta \mathrm{T}$ cells were significantly higher in the Immunity_H subtype in both GEO datasets as shown in Fig. S1B.

The prognostic values of immune subtype were analyzed by survival analysis. Compared to the Immunity_L, the Immunity_H subtype had a significantly higher survival in GSE29272 (log-rank $P<0.001)$. Although

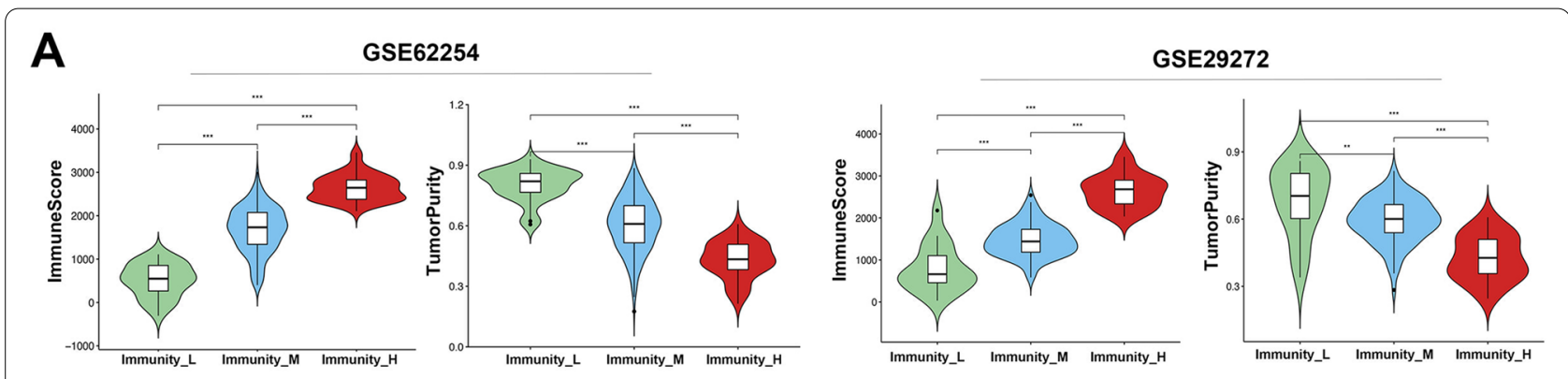

B

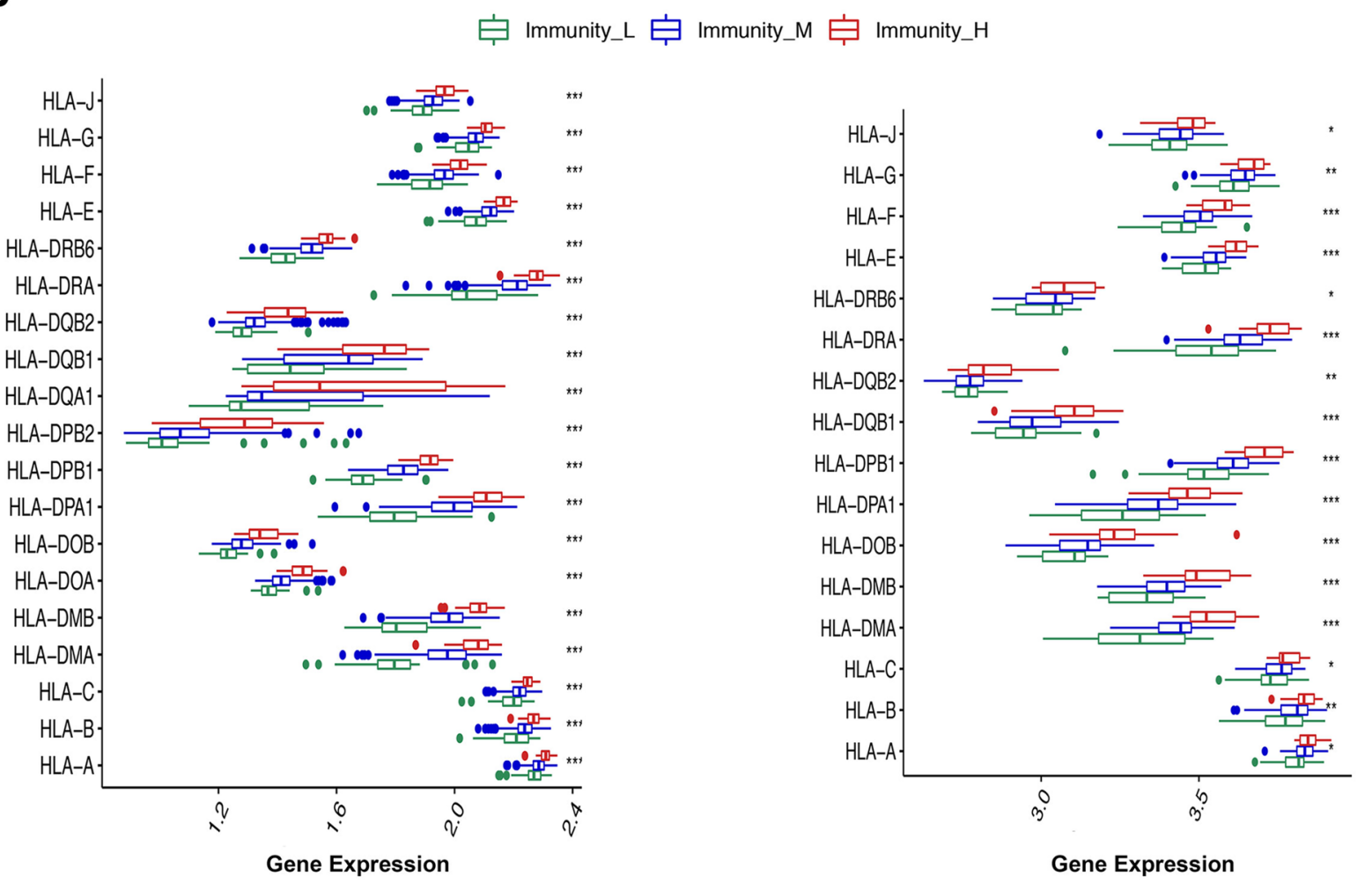

Fig. 2 Characteristics of different immune-related subtypes. A The level of immune cell infiltration in different immune subtypes (MannWhitney $U$ test). B Comparison of HLA genes expression between different immune subtypes (ANOVA test) 


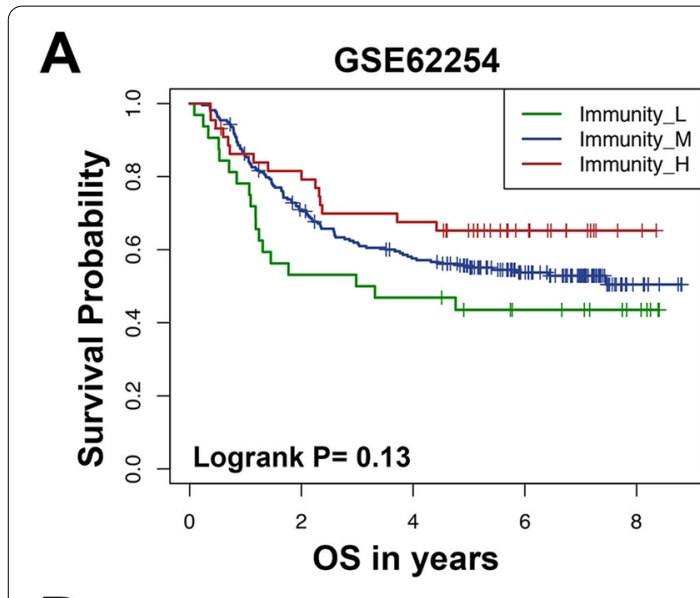

B

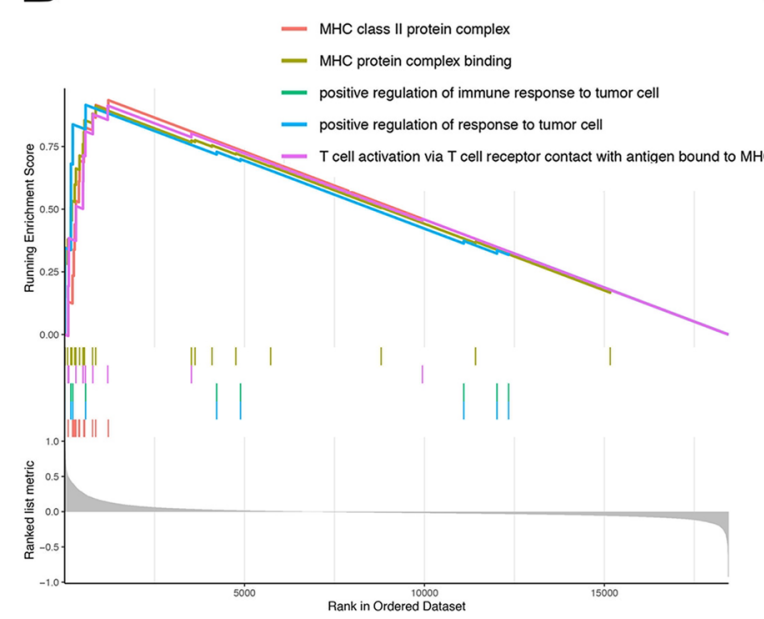

C
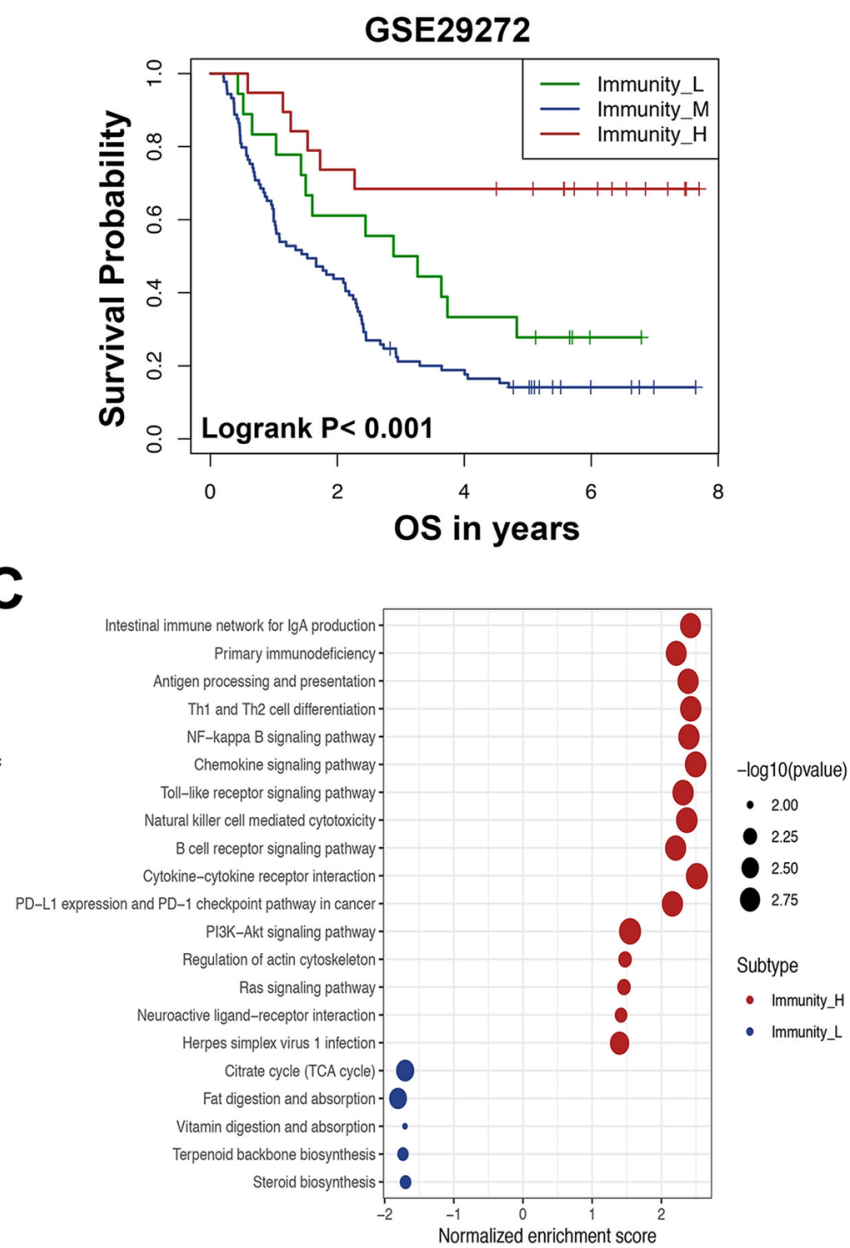

D
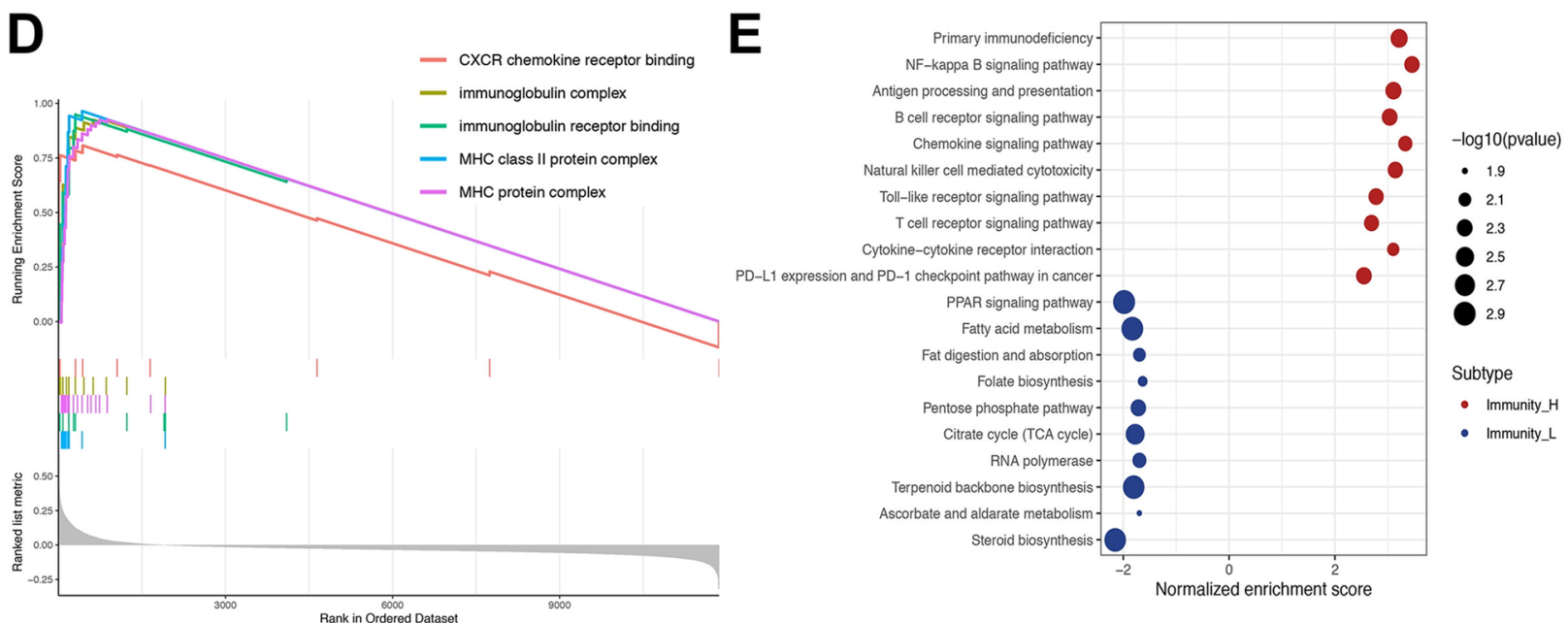

Fig. 3 Gene set enrichment analysis of immune-related subtypes. A Overall survival of different immune subtypes by Kaplan-Meier analysis (log-rank test $P=0.13$ in GSE62254; $P<0.001$ in GSE29272). B GSEA GO analysis of Immunity_H subtypes in GSE62254. C KEGG enrichment analysis of Immunity_H and Immunity_L subtypes in GSE62254. D GSEA GO analysis of Immunity_H subtypes in GSE29272. E KEGG enrichment analysis of Immunity_H and Immunity_L subtypes in GSE29272 
there has no statistical significance in GSE62254 (logrank $P=0.13)$, the Immunity_H still showed improved survival outcome compared to the Immunity_M and Immunity_L subtypes (Fig. 3A). To further confirm the prognostic value of Immuntiy_H subtype, we analyzed the prognostic difference between Immunity_H and Immunity_L subtypes in two independent datasets. As show in Fig. S1C, the Immunity_H presents significantly better OS compare to Immunity_L both in GSE62254 (log-rank $P=0.047)$ and GSE29272(log-rank $P<0.001)$. These findings showed that high immune activity could be associated with better clinical outcomes in AGC.

\section{Gene Set Enrichment Analysis (GSEA) of immune subtypes} To explore the potential mechanism participating in high immune activity subtype in AGC, a number of GO terms and KEGG pathways were identified in Immuntiy_H by GSEA. In GSE62254, the MHC class II protein complex, the $\mathrm{MHC}$ protein complex binding, positive regulation of immune response to tumor cells and positive regulation of response to tumor cells were the most significantly enriched cellular functions (Fig. 3B). CXCR chemokine receptor binding, the immunoglobulin complex, the immunoglobulin receptor binding, the MHC class II protein complex and the MHC protein complex were significantly enriched in GSE29272 (Fig. 3D). KEGG pathway enrichment analysis indicated that immune-associated pathways were highly enriched in the Immunity_H subgroup in both GSE62254 and GSE29272, which included primary immunodeficiency, antigen processing and presentation, the $\mathrm{B}$ cell receptor signaling pathway, chemokine signaling, natural killer cell-mediated cytotoxicity, the Toll-like receptor signaling pathway, PD-L1 expression and the PD-1 checkpoint pathway in cancer (Fig. 3C, E). These results confirmed that immune activity was up-regulate in the Immuntiy_H subtype. In contrast, citrate cycle, fat digestion and absorption and steroid biosynthesis were enriched in the Immunity_L subgroup suggesting that these pathways could be negatively correlated with immune activity in AGC.

\section{Association between immune subtypes and traditional classification}

We compared the immune subtypes and traditional classification of GC in GSE62254. The results showed that
Immunity_H was highly correlated with MSI (Fisher's exact test, $p=0.022$ ) and Lauren diffuse subtype (Fisher's exact test, $p<0.001$ ) (Fig. $4 \mathrm{~A}$ ). In the Immunity_L subgroup, MSS and the Lauren intestinal subtypes were mostly detected. Recent studies have indicated that MSI was associated with immunotherapeutic response in GC [6]. In addition, non-intestinal histology by Lauren classification was associated with higher degree of host immune response [26].

\section{Identification of vital candidate markers of immune subtypes}

To further investigate candidate markers associated with immune activity in AGC, we constructed a gene co-expression network of the GSE62254 dataset by WCGNA. In the process of constructing the network, the power value is a vital parameter that can affect the independence and average connectivity degree of the co-expression modules. In order to make the co-expression network approximate to scale-free topology distribution, the soft-thresholding power equals 3 was selected (Fig. 4B, Fig. S1D). Among of 16 modules, four pairs of gene modules were merged with high adjacency degree base on the threshold 0.2(Fig. S1E) and a total of 12 modules were identified to further analysis. Correlation between immune subtypes and module eigengene was analyzed, which shows the brown, midnight blue, tan and purple modules were positively associated with immune status. In contrast, the black, magenta, yellow and greenyellow modules were negatively associated with immune traits (Fig. 4C). To identify the critical genes for immune subtypes in AGC, the brown module that was the most strongly correlated module to the Immunity_H was selected (Fig. 4D, E). A protein-protein network (PPI) of the brown module was constructed consisting of 955 nodes and 248,141 edges. Parameters of degree, betweenness and closeness were used to describe the topological features of the PPI network. Genes with degree $>750$ (2 folds of median value 375), betweenness $>5.7 \mathrm{e}-6$ (Median value) and closeness $>0.622$ (Median value) were selected to construct the hub-network including 275 nodes and 37,675 edges (Fig. 5A) (Table S2). The Molecular Complex Detection (MCODE) was applied to screen the hub-cluster. Interestingly, the top significant cluster was consistent with the hub-network, indicating that these 275 genes were highly correlated with the Immunity_H subtype.

\footnotetext{
(See figure on next page.)

Fig. 4 Construction of co-expression network of immune-related subtypes. A Comparison of the immune-related classification and traditional classification of gastric cancer in GSE62254 and Immunity_H was highly correlated with MSI (Fisher's exact test, $p=0.022$ ) and lauren diffuse subtype (Fisher's exact test, $p<0.001$ ). B The correlation of different soft threshold power values of co-expression network. $\mathbf{C}$ Module-trait associations were evaluated by correlations between module eigengene and clinical traits. D The correlation of genes in brown module with immune subtypes trait. E The correlation of genes in magenta module with immune subtypes trait
} 


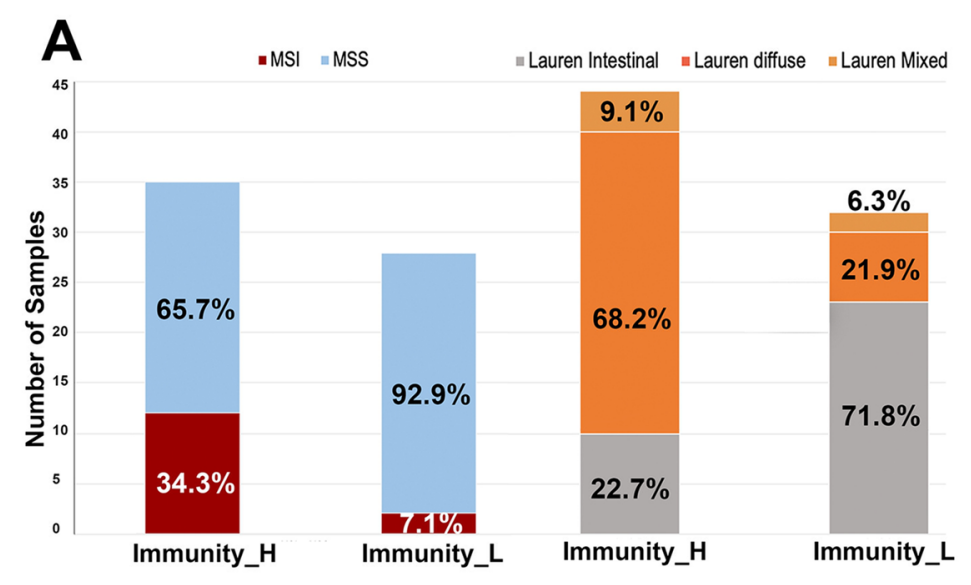

C

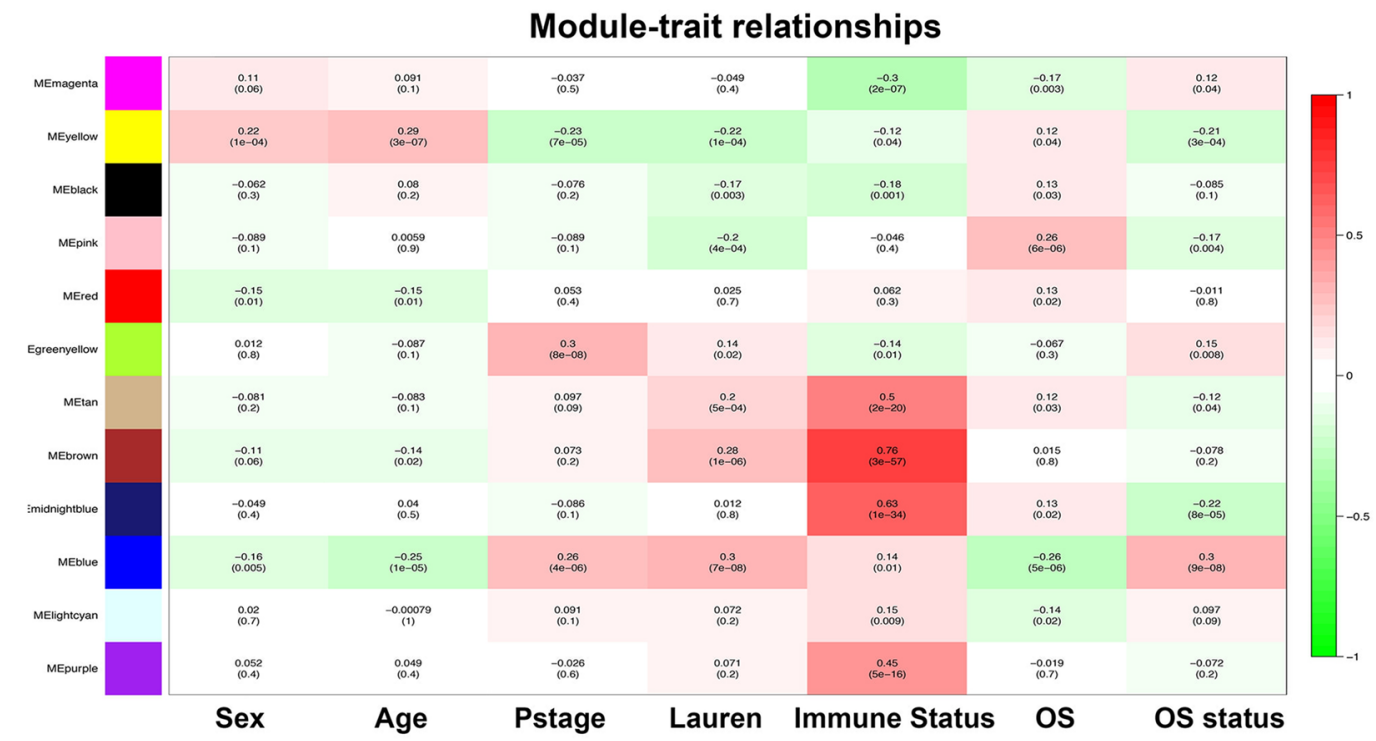

D

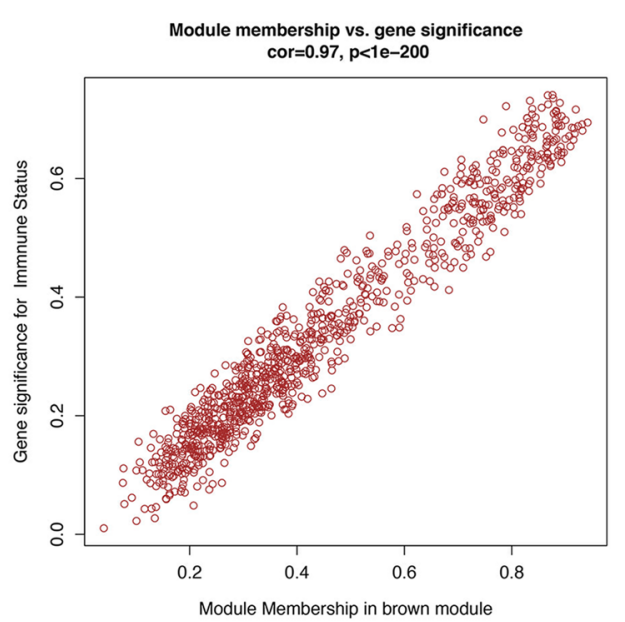

B

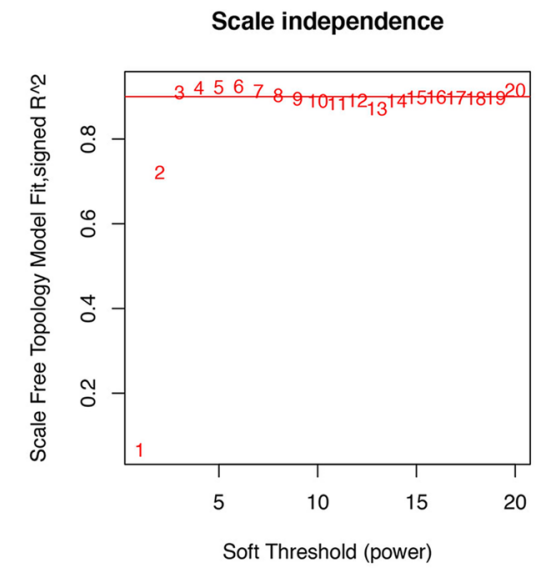


Furthermore, ssGSEA score was recalculated base on 275-gene signature and the cluster heatmap showed high expression of 275 hub-genes are strongly correlated with Imunity_H subtype and high immune cell infiltration (Fig. S2). The enrichment analysis of biological processes of 275 genes showed that immune-associated processes were significantly enriched (Fig. 5B). Among hub-gene network, the gene which have the highest Neighborhood Connectivity, Degree and MCODE score was confirmed to be the seed gene. ADAMDEC1 was selected to further analysis as the seed gene in 275 hub-genes (Table S3). Kaplan-Meier analysis of overall survival (OS) demonstrated that high levels of ADAMDEC1 were significantly associated with better prognosis in both GSE62254 (log-rank $P=0.008$ ) and external validation of GSE29272 (log-rank $P=0.023)$ and KMplotter (log-rank $P=0.031$ ) (Fig. 5C).

\section{The role of ADAMDEC1 in the prediction of immunotherapeutic response}

In order to investigate the value of ADAMDEC1 in speculating the therapeutic response, the samples who received immunotherapy in the IMvigor210 cohort were selected to further analysis. In a total of 298 urothelial cancer samples, high level of ADAMDEC1 expression showed significant better OS compared to low expression group (log-rank $P=0.01)$. We also found that high expression of ADAMDEC1 was correlated with objective response to anti-PDL1 therapy (Fisher's exact test, $p=0.031$ ) (Fig. 5D-E). These findings suggested the predictive value of ADAMDEC1.

\section{Experimental validation by gastric cancer cells}

To further confirm the potential function of ADAMDEC1 in MGC803 gastric cancer cell line, silencing of ADAMDEC1 expression by siRNA was conducted (Fig. 6A). The results demonstrated that deficiency of ADAMDEC1 promoted gastric cancer cell proliferation and migration as presented in Fig. 6B, C. Interestingly, PD-L1 mRNA and expression levels were both upregulated following with depletion of ADAMDEC1 in MGC803 cell line (Fig. 6D, E). Besides, apoptosis in Jurkat $\mathrm{T}$ cells was enhanced significantly after co-incubated with ADAMDEC1 silencing MGC803 cell (Fig. 6F). These findings suggested that ADAMDEC1 was a critical marker in predicting proliferation and immune response in gastric cancer cells.

\section{Discussion}

AGC remains a major clinical problem with poor prognosis due to the limited effectiveness of therapies. Although immune checkpoint blockades provide a treatment paradigm, only a small number of patients may benefit from treatment. Current classifications of GC are mostly based on genomic analysis or molecular features. Recently, studies have focused on GC classification based on immune profiling [27-29]. In the present study, GC was classified into three immune-related subtypes, which included Immunity_H, Immunity_M and Immunity_L. The immunity high subtype was positively correlated with immune score and negatively correlated with tumor purity, which showed that Immunity_H cancers were strongly infiltrated by immune cells and had high immune activities. Immunity_H also indicated higher immunogenicity compared to the other subtypes because of high expression of HLA genes. In addition, the proportions of 22 immune gene signatures were calculated by CIBRTSORT. Macrophages $\mathrm{M} 1, \mathrm{CD} 4 \mathrm{~T}$ cells, CD8 $\mathrm{T}$ cells and $\gamma \delta \mathrm{T}$ cells were both highly presented in the Immunity_H subtype of the two independent datasets which further suggested increased anti-tumor immune activity in the immunity_H subgroup. Survival analysis confirmed favorable prognosis of the Immunity_H subtype in GSE29272. Whilst there was no significant difference in overall survival between the subtypes in GSE62254, the survival curve showed the same trend in GSE29272. These results are consistent with numerous previous studies which have showed better prognosis in the high immune cell infiltration group $[13,30]$.

Gene ontology analysis showed that the MHC terms were enriched in Immunity_H subtype of both GSE datasets. Furthermore, we found that the immunity high subtype was highly enriched in immune signatures such as primary immunodeficiency, antigen processing and presentation, B cell receptor signaling pathway, PD-L1 expression and PD-1 checkpoint pathway in cancer. In addition, cancer-associated pathways including PI3K-AKT and RAS signaling were also enriched in the immunity high subtype. Previous studies showed that PI3K-AKT and RAS signaling pathways have participated in multiple immunity processes in the tumor [31-33], suggesting the potential roles of these pathways in regulating immune

\footnotetext{
(See figure on next page.)

Fig. 5 Identification of the hub-network and hub-genes in Immunity_H subtype. A PPI network of genes in brown module which including 955 nodes and 248,141 edges. Hub-network was extracted from PPI network according to topological features and MCODE algorithm which consist of 275 nodes and 37,675 edges. B Significantly enriched KEGG pathways of hub-network genes. C Overall Survival (OS) of ADAMDEC1 in GSE62254 cohort by Kaplan-Meier (KM) analysis (log-rank $P<0.008)$ and external validation by GSE29272 and KMplotter cohort (log-rank $P=0.023, P=0.031$, respectively). D Overall survival of high and low ADAMDEC1 expression by Kaplan-Meier analysis in IMvigor210 cohort (Log rank test, $p=0.01)$. $\mathbf{E}$ Rate of clinical response to anti-PD-L1 immunotherapy in high and low ADAMDEC1 expression groups in IMvigor210 cohort
} 


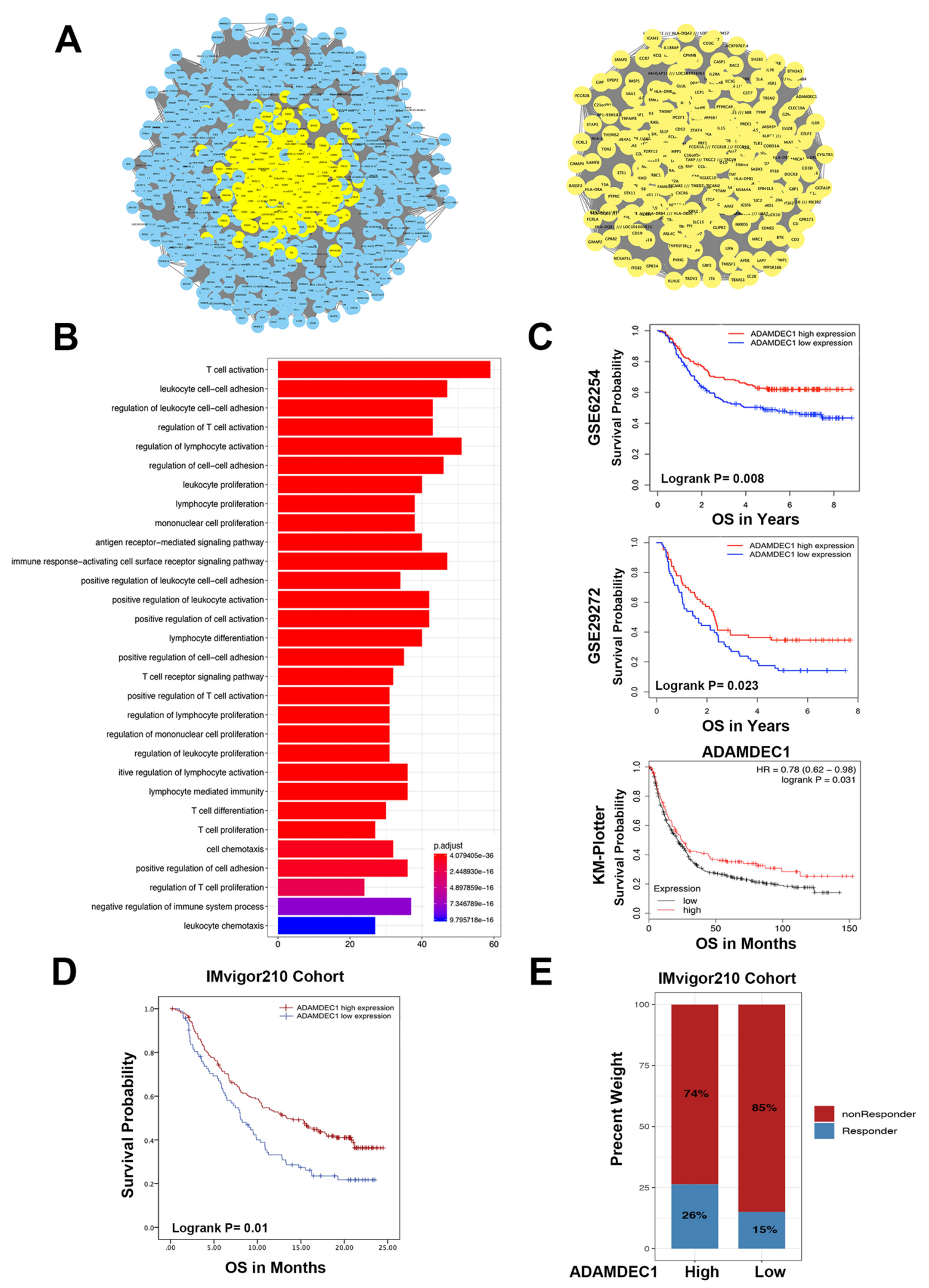

Fig. 5 (See legend on previous page.) 


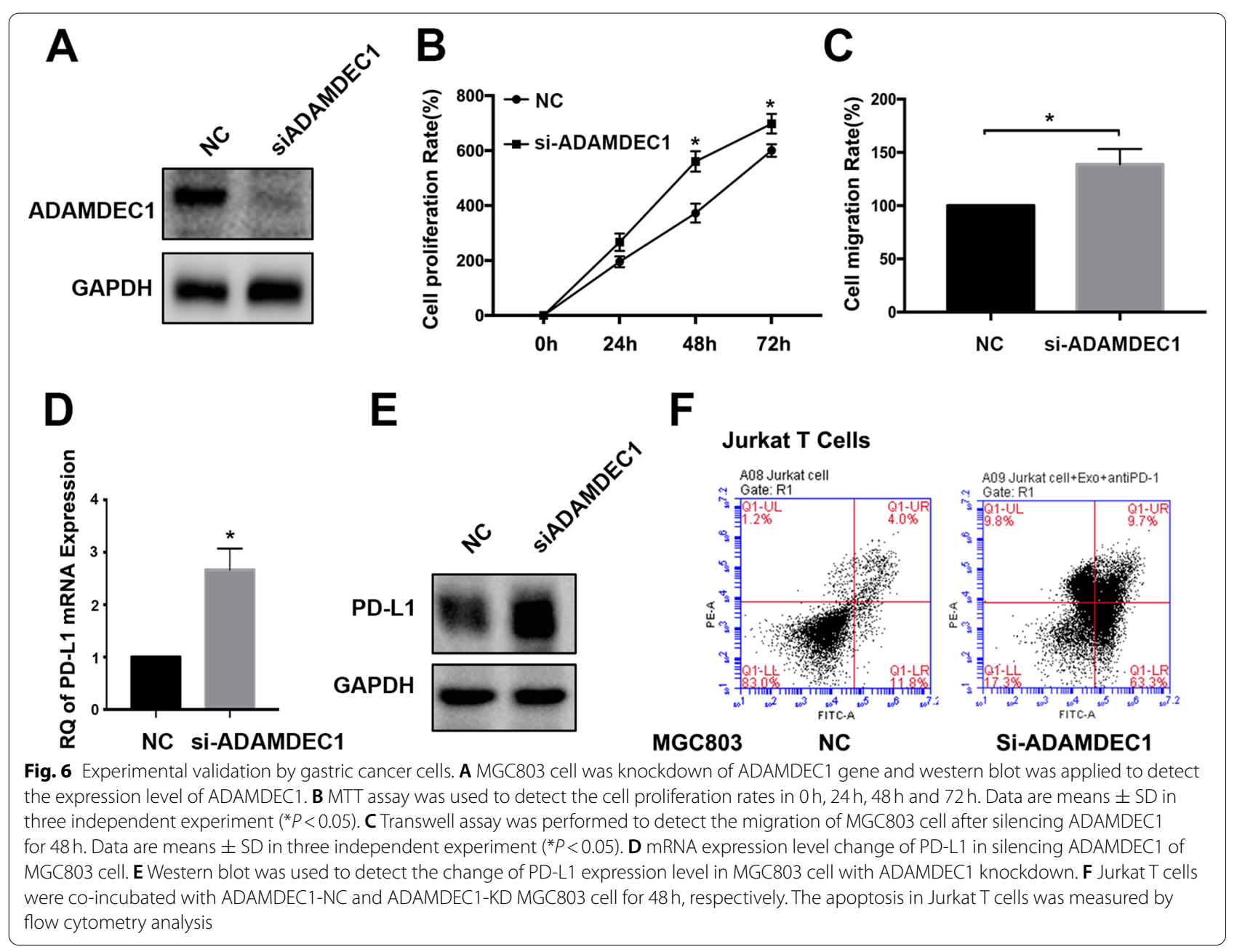

activity in AGC. In contrast, immune-related pathways were not frequently enriched in the Immunity_L subtype.

Comparison of immune subtypes and traditional classification in GC indicated that the Immunity_H subtype was associated with MSI and the lauren diffuse type. MSI has been confirmed to be part of the sensitive index to anti-PD-1/PD-L1 treatment. Thus, these findings suggest that the Immunity_H subtype of AGC might benefit from immunotherapy.

To further identify the critical markers of the Immunity_H subtype, we constructed a co-expression network of the GSE62254 dataset by WGCNA. The Brown module was mostly correlated with the Immunity_H subtype. The hub-network was constructed by calculating the topological features of the PPI network of the brown module. GO analysis of the hub-network genes indicated that immuneassociated biological processes were highly enriched which are represented by $\mathrm{T}$ cell activation and immune response. Based on the MCODE algorithm, 275 genes within the hubnetwork contributed equally to the network and accurately represented the Immunity_H subtype. Among the 275 genes, ADAMDEC1 was marked as seed gene.

ADAM like decysin 1 (ADAMDEC1) is a member of the ADAM family of metalloproteinases. Unlike the other ADAM family member, ADAMDEC1 lack of a transmembrane domain and altered catalytic domain [34, 35]. In the current study, ADAMDEC1 was confirmed to be associated with favorable prognosis in gastric cancer. Several studies have shown that ADAMDEC1 as a prognostic factor in gastric adenocarcinoma and the mRNA expression of ADAMDEC1 is decreased during both tumorigenesis and tumor progression in colorectal cancer [36, 37]. In vitro, ADAMDEC1 could negatively regulate GC cells proliferation and migration. In addition, we found that depletion of ADAMDEC1 increased PD-L1 expression in gastric cancer cell. A systematic review and meta-analysis confirmed that PD-L1 overexpression is a significant adverse prognostic factor in gastric cancer [38]. Besides, ADAMDEC1 has been reported to function in regulating the immune response and might play an important role in dendritic cell 
function [35]. The patients who receiving immunotherapy were evaluated by IMvigor210 datasets as the independent validation [39], we notice that the expression level of ADAMDEC1 was significantly upregulated in patients responding to immunotherapy and survival benefit also detected in high ADAMDEC1 group. Furthermore, silencing ADAMDEC1 of gastric cancer cells promoted apoptosis of Jurkat $\mathrm{T}$ cells. Collectively, ADAMDEC might serve as biomarker of prognosis and immune response in AGC.

\section{Conclusions}

In summary, classification based on immune signatures reflected immune activity in different subtypes in AGC. ADAMDEC1 served as hub-gene was identified and validated to confirm predictive values in immune activity and prognostic values in AGC patients. The mechanism regulating the TME and clinical application value of ADAMDEC1 require further investigation. This study can potentially provide novel biomarker and therapeutic targets in AGC.

\begin{abstract}
Abbreviations
AGC: Advanced gastric cancer; ssGSEA: Single-sample gene-set enrichment analysis; WGCNA: Weighted gene co-expression network analysis; MCODE: Molecular Complex Detection; TME: Tumor immune microenvironment; ESTIMATE: Estimation of Stromal and Immune Cells in Malignant Tumors using Expression data; GEO: Gene Expression Omnibus; GO: Gene Ontology; KEGG: Kyoto Encyclopedia of Genes and Genomes; ADAMDEC1: ADAM like decysin 1.
\end{abstract}

\section{Supplementary Information}

The online version contains supplementary material available at https://doi. org/10.1186/s12885-021-09065-z.

\begin{abstract}
Additional file 1: Supplementary Figure 1. (A) The level of Stromal score in different immune subtypes (MannWhitney $U$ test). (B) Comparison of the fraction of immune cell subtypes between different immune subtypes (Kruskal-Wallis test). ${ }^{*} P<0.05 ;{ }^{*} P<0.01 ;{ }^{* *} P<0.001$. (C) Overall survival of Immunity_H and Immunity_L subtypes by Kaplan-Meier analysis (log-rank test $P=0.047$ in GSE62254; $P<0.001$ in GSE29272). (D) Identification of the best soft-thresholding power value. (E) 4 pairs of gene modules were merged due to their similarity according to the threshold (Red line). (F) The gene dendrogram was constructed by hierarchical clustering base on dissTOM of genes. (G) Heatmap plot of the adjacencies of modules. Supplementary Figure 2. (A, B) High expression level of 275 hub-genes was correlated with Immunity_H subtype and high immune cell infiltration. ***P $<0.001$.

Additional file 2.

Additional file 3.

Additional file 4.
\end{abstract}

\section{Acknowledgements}

The authors would like to acknowledge the Key Laboratory of Precision Diagnosis and Treatment of Gastrointestinal Tumors, Ministry of Education (China Medical University, Shenyang, China) for providing the space and equipment for conducting the experiments.

\section{Authors' contributions}

SMZ, XJQ contribution to conception and design. MZL Cell experiments. YC Data acquisition. SW, CL. Data analysis and interpretation. All authors read and approved the final manuscript.

\section{Funding}

The present study was supported by The National Key Research and Development Program of China (2017YFC1308900); National Natural Science Foundation of China (NO.81803092); Technological Special Project of Liaoning Province of China (2019020176-JH1/103); Science and Technology Plan Project of Liaoning Province (NO.2013225585); The General Projects of Liaoning Province Colleges and Universities (LFWK201706).

\section{Availability of data and materials}

The datasets used and/or analysed during the current study are available in TCGA (https://portal.gdc.cancer.gov) and GEO datasets (GSE62254: https:// www.ncbi.nlm.nih.gov/geo/query/acc.cgi?acc=GSE62254; GSE29272: https:// www.ncbi.n/m.nih.gov/geo/query/acc.cgi?acc=GSE29272) and IMvigor210 datasets: http://research-pub.gene.com/IMvigor210CoreBiologies.

\section{Declarations}

Ethics approval and consent to participate

Data retrieved from the GEO controlled-access database was collected using tumors from patients who provided informed consent based on guidelines laid out by the GEO Ethics, Law and Policy Group.

\section{Consent for publication}

Not applicable.

\section{Competing interests}

The authors declare that they have no competing interests.

\section{Author details}

${ }^{1}$ Department of Medical Oncology, the First Hospital of China Medical University, 110001 Shenyang, China. ${ }^{2}$ Key Laboratory of Anticancer Drugs and Biotherapy of Liaoning Province, the First Hospital of China Medical University, Shenyang 110001, China. ${ }^{3}$ Liaoning Province Clinical Research Center for Cancer, Shenyang 110001, China. ${ }^{4}$ Key Laboratory of Precision Diagnosis and Treatment of Gastrointestinal Tumors, Ministry of Education, Shenyang 110001, China. ${ }^{5}$ Department of Plastic Surgery, the First Hospital of China Medical University, Shenyang 110001, China.

Received: 28 May 2021 Accepted: 25 November 2021

Published online: 11 December 2021

\section{References}

1. Bray F, Ferlay J, Soerjomataram I, Siegel RL, Torre LA, Jemal A. Global cancer statistics 2018: GLOBOCAN estimates of incidence and mortality worldwide for 36 cancers in 185 countries. CA Cancer J Clin. 2018;68(6):394-424.

2. Digklia A, Wagner AD. Advanced gastric cancer: current treatment landscape and future perspectives. World J Gastroenterol. 2016;22(8):2403-14.

3. Larkin J, Chiarion-Sileni V, Gonzalez R, Grob JJ, Cowey CL, Lao CD, et al. Combined Nivolumab and Ipilimumab or Monotherapy in untreated melanoma. N Engl J Med. 2015;373(1):23-34

4. Forde PM, Chaft JE, Smith KN, Anagnostou V, Cottrell TR, Hellmann MD, et al. Neoadjuvant PD-1 blockade in Resectable lung Cancer. N Engl J Med. 2018;378(21):1976-86.

5. Fuchs CS, Doi T, Jang RW, Muro K, Satoh T, Machado M, et al. Safety and efficacy of Pembrolizumab Monotherapy in patients with previously treated advanced gastric and Gastroesophageal junction Cancer: phase 2 clinical KEYNOTE-059 trial. JAMA Oncol. 2018;4(5):e180013.

6. Le DT, Durham JN, Smith KN, Wang H, Bartlett BR, Aulakh LK, et al. Mismatch repair deficiency predicts response of solid tumors to PD-1 blockade. Science (New York, NY). 2017;357(6349):409-13.

7. Taube JM, Klein A, Brahmer JR, Xu H, Pan X, Kim JH, et al. Association of PD-1, PD-1 ligands, and other features of the tumor immune microenvironment with response to anti-PD-1 therapy. Clin Cancer Res. 2014;20(19):5064-74.

8. Binnewies M, Roberts EW, Kersten $\mathrm{K}$, Chan V, Fearon DF, Merad M, et al. Understanding the tumor immune microenvironment (TIME) for effective therapy. Nat Med. 2018;24(5):541-50. 
9. Tekpli X, Lien T, Rossevold AH, Nebdal D, Borgen E, Ohnstad HO, et al. An independent poor-prognosis subtype of breast cancer defined by a distinct tumor immune microenvironment. Nat Commun. 2019;10(1):5499.

10. Lazar DC, Avram MF, Romosan I, Cornianu M, Taban S, Goldis A. Prognostic significance of tumor immune microenvironment and immunotherapy: novel insights and future perspectives in gastric cancer. World J Gastroenterol. 2018;24(32):3583-616.

11. Balch CM, Riley LB, Bae YJ, Salmeron MA, Platsoucas CD, von Eschenbach A, et al. Patterns of human tumor-infiltrating lymphocytes in 120 human cancers. Arch Surg. 1990;125(2):200-5.

12. Caruso RA, Bellocco R, Pagano M, Bertoli G, Rigoli L, Inferrera C. Prognostic value of intratumoral neutrophils in advanced gastric carcinoma in a high-risk area in northern Italy. Mod Pathol. 2002;15(8):831-7.

13. Jiang W, Liu K, Guo Q, Cheng J, Shen L, Cao Y, et al. Tumor-infiltrating immune cells and prognosis in gastric cancer: a systematic review and meta-analysis. Oncotarget. 2017:8(37):62312-29.

14. Yoshihara K, Shahmoradgoli M, Martinez E, Vegesna R, Kim H, TorresGarcia W, et al. Inferring tumour purity and stromal and immune cell admixture from expression data. Nat Commun. 2013;4:2612.

15. Alonso MH, Ausso S, Lopez-Doriga A, Cordero D, Guino E, Sole X, et al. Comprehensive analysis of copy number aberrations in microsatellite stable colon cancer in view of stromal component. Br J Cancer. 2017;117(3):421-31.

16. Xu M, Li Y, Li W, Zhao Q, Zhang Q, Le K, et al. Immune and Stroma related genes in breast Cancer: a comprehensive analysis of tumor microenvironment based on the Cancer genome atlas (TCGA) database. Front Med. 2020;7:64.

17. Yan H, Qu J, Cao W, Liu Y, Zheng G, Zhang E, et al. Identification of prognostic genes in the acute myeloid leukemia immune microenvironment based on TCGA data analysis. Cancer Immunol Immunother. 2019:68(12):1971-8

18. Hanzelmann S, Castelo R, Guinney J. GSVA: gene set variation analysis for microarray and RNA-seq data. BMC Bioinformatics. 2013;14:7.

19. He Y, Jiang Z, Chen C, Wang X. Classification of triple-negative breast cancers based on Immunogenomic profiling. J Exp Clin Cancer Res. 2018;37(1):327.

20. Newman AM, Liu CL, Green MR, Gentles AJ, Feng W, Xu Y, et al. Robust enumeration of cell subsets from tissue expression profiles. Nat Methods. 2015;12(5):453-7.

21. Langfelder P, Horvath S. WGCNA: an R package for weighted correlation network analysis. BMC Bioinformatics. 2008;9:559.

22. Yu G, Wang LG, Han Y, He QY. clusterProfiler: an $R$ package for comparing biological themes among gene clusters. Omics. 2012;16(5):284-7.

23. Shannon P, Markiel A, Ozier O, Baliga NS, Wang JT, Ramage D, et al. Cytoscape: a software environment for integrated models of biomolecular interaction networks. Genome Res. 2003;13(11):2498-504.

24. Bader GD, Hogue CW. An automated method for finding molecular complexes in large protein interaction networks. BMC Bioinformatics. 2003;4:2.

25. Zhang S, Zang D, Cheng Y, Li Z, Yang B, Guo T, et al. Identification of key gene and Pathways for the prediction of peritoneal metastasis of gastric Cancer by co-expression analysis. J Cancer. 2020;1 1(10):3041-51.

26. Byeon SJ, Heo YJ, Cho J, An JY, Choi MG, Lee JH, et al. Factors associated with host immune response and number of lymph nodes: a large retrospective cohort study. Ann Surg Oncol. 2018;25(12):3621-8.

27. Zhou YJ, Zhu GQ, Lu XF, Zheng KI, Wang QW, Chen JN, et al. Identification and validation of tumour microenvironment-based immune molecular subgroups for gastric cancer: immunotherapeutic implications. Cancer Immunol Immunother. 2020;69:1057-69.

28. Jiang Z, Liu Z, Li M, Chen C, Wang X. Immunogenomics analysis reveals that TP53 mutations inhibit tumor immunity in gastric Cancer. Transl Oncol. 2018;11(5):1171-87.

29. Zeng D, Li M, Zhou R, Zhang J, Sun H, Shi M, et al. Tumor microenvironment characterization in gastric Cancer identifies prognostic and Immunotherapeutically relevant gene signatures. Cancer Immunol Res. 2019;7(5):737-50.

30. Ali HR, Provenzano E, Dawson SJ, Blows FM, Liu B, Shah M, et al. Association between CD8+T-cell infiltration and breast cancer survival in 12,439 patients. Ann Oncol. 2014;25(8):1536-43.
31. Coelho MA, de Carne TS, Rana S, Zecchin D, Moore C, Molina-Arcas M, et al. Oncogenic RAS signaling promotes tumor Immunoresistance by stabilizing PD-L1 mRNA. Immunity. 2017;47(6):1083-1099.e1086.

32. Noh KH, Kang TH, Kim JH, Pai SI, Lin KY, Hung CF, et al. Activation of Akt as a mechanism for tumor immune evasion. Mol Ther. 2009;17(3):439-47.

33. Rogel A, Willoughby JE, Buchan SL, Leonard HJ, Thirdborough SM, Al-Shamkhani A. Akt signaling is critical for memory CD8(+) T-cell development and tumor immune surveillance. Proc Natl Acad Sci U S A. 2017:114(7):E1178-e1187.

34. Jimenez-Pascual A, Hale JS, Kordowski A, Pugh J, Silver DJ, Bayik D, et al. ADAMDEC1 maintains a growth factor signaling loop in Cancer stem cells. Cancer Discov. 2019:9(11):1574-89.

35. Bates EE, Fridman WH, Mueller CG. The ADAMDEC1 (decysin) gene structure: evolution by duplication in a metalloprotease gene cluster on chromosome 8p12. Immunogenetics. 2002;54(2):96-105.

36. Macartney-Coxson DP, Hood KA, Shi HJ, Ward T, Wiles A, O'Connor R, et al. Metastatic susceptibility locus, an $8 p$ hot-spot for tumour progression disrupted in colorectal liver metastases: 13 candidate genes examined at the DNA, mRNA and protein level. BMC Cancer. 2008;8:187.

37. Pasini FS, Zilberstein B, Snitcovsky I, Roela RA, Mangone FR, Ribeiro U Jr, et al. A gene expression profile related to immune dampening in the tumor microenvironment is associated with poor prognosis in gastric adenocarcinoma. J Gastroenterol. 2014;49(11):1453-66.

38. Gu L, Chen M, Guo D, Zhu H, Zhang W, Pan J, et al. PD-L1 and gastric cancer prognosis: a systematic review and meta-analysis. PLoS One. 2017;12(8):e0182692.

39. Zhang X, Shi M, Chen T, Zhang B. Characterization of the immune cell infiltration landscape in head and neck squamous cell carcinoma to aid immunotherapy. Mol Ther Nucleic Acids. 2020;22:298-309.

\section{Publisher's Note}

Springer Nature remains neutral with regard to jurisdictional claims in published maps and institutional affiliations.

Ready to submit your research? Choose BMC and benefit from:

- fast, convenient online submission

- thorough peer review by experienced researchers in your field

- rapid publication on acceptance

- support for research data, including large and complex data types

- gold Open Access which fosters wider collaboration and increased citations

- maximum visibility for your research: over $100 \mathrm{M}$ website views per year

At BMC, research is always in progress.

Learn more biomedcentral.com/submissions 